\title{
STIT Tessellations have trivial tail $\sigma$-algebra
}

\author{
S. Martínez \\ Departamento Ingeniería Matemática and Centro Modelamiento Matemático, \\ Universidad de Chile, \\ UMI 2807 CNRS, Casilla 170-3, Correo 3, Santiago, Chile. \\ Email: smartine@dim.uchile.cl \\ Werner Nagel \\ Friedrich-Schiller-Universität Jena, \\ Institut für Stochastik, \\ Ernst-Abbe-Platz 2, D-07743 Jena, Germany. \\ Email: werner.nagel@uni-jena.de
}

\begin{abstract}
We consider homogeneous STIT tessellations $Y$ in the $\ell$-dimensional Euclidean space $\mathbb{R}^{\ell}$ and show the triviality of the tail $\sigma$-algebra. This is a sharpening of the mixing result by Lachièze-Rey 8 .
\end{abstract}

Keywords: Stochastic geometry; Random process of tessellations; Ergodic theory; tail $\sigma$-algebra

AMS subject classification 60D0560J25, 60J75, 37A25

\section{Introduction}

Let $Y=\left(Y_{t}: t>0\right)$ be the STIT tessellation process, which is a Markov process taking values in the space of tessellations of the $\ell$-dimensional Euclidean space $\mathbb{R}^{\ell}$. The process $Y$ is spatially stationary (that is its law is invariant under translations of the space) and on every polytope with nonempty interior $W$ (called a window) the induced tessellation process, denoted by $Y \wedge W=\left(Y_{t} \wedge W\right.$ : $t>0$ ), is a pure jump process. The process $Y$ was firstly constructed in $[12$ ] and in Section 2 we give a brief description of it and recall some of its main properties.

In stochastic geometry, ergodic and mixing properties as well as weak dependencies in space are studied. E.g., Heinrich et al. considered mixing properties for Voronoi and some other tessellations, Poisson cluster processes and germgrain models and derived Laws of Large Numbers and Central Limit Theorems, see [3, 6, 5].

For STIT tessellations, Lachièze-Rey [8] showed that they are mixing. Schreiber and Thäle [15, 16, 17] proved some limit theorems. They provided Central 
Limit Theorems for the number of vertices and the total edge length in the twodimensional case $(\ell=2)$. Furthermore, they proved that in dimensions $\ell \geq 3$ there appear non-normal limits, e.g. for the total surface area of the cells of STIT tessellations.

An issue is the problem of triviality of the tail $\sigma$-algebras for certain class of distributions. For random measures and point processes a key reference is the book by Daley and Vere-Jones ([2], pp. 207-209).

In Section 3 of the present paper we introduce a definition for the tail $\sigma$ algebra $\mathcal{B}_{-\infty}(\mathbb{T})$ on the space $\mathbb{T}$ of tessellations in the $\ell$-dimensional Euclidean space $\mathbb{R}^{\ell}$. This definition relies essentially on the definition of the Borel $\sigma$ algebra with respect to the Fell topology on the set of closed subsets of $\mathbb{R}^{\ell}$ (cf. [14]) as well as on the mentioned definition of the tail $\sigma$-algebra for random measures and point processes, as given in 2.

Our main result is formulated in in Section 4, Theorem 2 2 For the distribution of a STIT tessellation, the tail $\sigma$-algebra $\mathcal{B}_{-\infty}(\mathbb{T})$ is trivial, i.e. all its elements (the terminal events) have either probability 1 or 0 . A detailed proof is given in Section 5

Finally, we compare STIT tessellations with Poisson hyperplane tessellations (PHT), and we show, that the tail $\sigma$-algebra $\mathcal{B}_{-\infty}(\mathbb{T})$ is not trivial with respect to the distribution of PHT.

\section{The STIT model}

\subsection{A construction of STIT tessellations}

Let $\mathbb{R}^{\ell}$ be the $\ell$-dimensional Euclidean space, denote by $\mathbb{T}$ the space of tessellations of this space as defined in [14] (Ch. 10, Random Mosaics). A tessellation can be considered as a set $T$ of polytopes (the cells) with disjoint interiors and covering the Euclidean space, as well as a closed subset $\partial T$ which is the union of the cell boundaries. There is an obvious one-to-one relation between both ways of description of a tessellation, and their measurable structures can be related appropriately, see [14, 9].

A compact convex polytope $W$ with non-empty interior in $\mathbb{R}^{\ell}$, is called a window. We can consider tessellations of $W$ and denote the set of all those tessellations by $\mathbb{T} \wedge W$. If $T \in \mathbb{T}$ we denote by $T \wedge W$ the induced tessellation on $W$. Its boundary is defined by $\partial(T \wedge W)=(\partial T \cap W) \cup \partial W$.

In the present paper we will refer to the construction given in 12] in all detail (for an alternative but equivalent construction see [9]). On every window $W$ there exists $Y \wedge W=\left(Y_{t} \wedge W: t>0\right)$ a STIT tessellation process, it turns out to be a pure jump Markov process and hence has the strong Markov property (see [1, Proposition 15.25). Each marginal $Y_{t} \wedge W$ takes values in $\mathbb{T} \wedge W$. Furthermore, for any $t>0$ the law of $Y_{t}$ is consistent with respect to the windows, that is if $W^{\prime}$ and $W$ are windows such that $W^{\prime} \subseteq W$, then 
$\left(Y_{t} \wedge W\right) \wedge W^{\prime} \sim Y_{t} \wedge W^{\prime}$, where $\sim$ denotes the identity of distributions (for a proof see [12]). This yields the existence of a STIT tessellation $Y_{t}$ of $\mathbb{R}^{\ell}$ such that for all windows $W$ the law of $Y_{t} \wedge W$ coincides with the law of the construction in the window. A global construction for a STIT process was provided in 11. A STIT tessellation process $Y=\left(Y_{t}: t>0\right)$ is a Markov process and each marginal $Y_{t}$ takes values in $\mathbb{T}$.

Here, let us recall roughly the construction of $Y \wedge W$ done in [12].

Let $\Lambda$ be a (non-zero) measure on the space of hyperplanes $\mathcal{H}$ in $\mathbb{R}^{\ell}$. It is assumed that $\Lambda$ is translation invariant and possesses the following locally finiteness property:

$\Lambda([B])<\infty, \forall$ bounded sets $B \subset \mathbb{R}^{\ell}$, where $[B]=\{H \in \mathcal{H}: H \cap B \neq \emptyset\}$.

(Notation $\subset$ means strict inclusion and $\subseteq$ inclusion or equality). It is further assumed that the support set of $\Lambda$ is such that there is no line in $\mathbb{R}^{\ell}$ with the property that all hyperplanes of the support are parallel to it (in order to obtain a.s. bounded cells in the constructed tessellation, cf. [14, Theorem 10.3.2, which can also be applied to STIT tessellations).

The assumptions made on $\Lambda$ imply $0<\Lambda([W])<\infty$ for every window $W$. Denote by $\Lambda_{[W]}$ the restriction of $\Lambda$ to $[W]$ and by $\Lambda^{W}=\Lambda([W])^{-1} \Lambda_{[W]}$ the normalized probability measure. Let us take two independent families of independent random variables $D=\left(d_{n, m}: n, m \in \mathbb{N}\right)$ and $\tau=\left(\tau_{n, m}: n, m \in \mathbb{N}\right)$, where each $d_{n, m}$ has distribution $\Lambda^{W}$ and each $\tau_{n, m}$ is exponentially distributed with parameter $\Lambda([W])$.

- Even if for $t=0$ the STIT tessellation $Y_{0}$ is not defined in $\mathbb{R}^{\ell}$, we define $Y_{0} \wedge W=\{W\}$ the trivial tessellation for the window $W$. Its unique cell is denoted by $C^{1}=W$.

- Any extant cell has a random lifetime, and at the end of its lifetime it is divided by a random hyperplane. The lifetime of $W=C^{1}$ is $\tau_{1,1}$, and at that time it is divided by $d_{1,1}$ into two cells denoted by $C^{2}$ and $C^{3}$.

- Now, for any cell $C^{i}$ which is generated in the course of the construction, the random sequences $\left(d_{i, m}: m \in \mathbb{N}\right)$ and $\tau=\left(\tau_{i, m}: m \in \mathbb{N}\right)$ are used, and the following rejection method is applied:

- When the time $\tau_{i, 1}$ is elapsed, the random hyperplane $d_{i, 1}$ is thrown onto the window $W$. If it does not intersect $C^{i}$ then this hyperplane is rejected, and we continue until a number $z_{i}$, which is the first index $j$ for which a hyperplane $d_{i, j}$ intersects and thus divides $C^{i}$ into two cells $C^{l_{1}(i)}, C^{l_{2}(i)}$, that are called the successors of $C^{i}$. Note that this random number $z_{i}$ is finite a.s. Hence the lifetime of $C^{i}$ is a sum $\tau^{*}\left(C^{i}\right):=\sum_{m=1}^{z_{i}} \tau_{i, m}$. It is easy to check, see [12, that $\tau^{*}\left(C^{i}\right)$ is exponentially distributed with parameter $\Lambda\left(\left[C^{i}\right]\right)$. Note that $z_{1}=1$ and $\tau^{*}\left(C^{1}\right)=\tau_{1,1}$. 
- This procedure is performed for any extant cell independently. It starts in that moment when the cell is born by division of a larger (the predecessor) cell. In order to guarantee independence of the division processes for the individual cells, the successors of $C^{i}$ get indexes $l_{1}(i), l_{2}(i)$ in $\mathbb{N}$ that are different, and that can be chosen as the smallest numbers which were not yet used before for other cells.

- For each cell $C^{i}$ we denote by $\eta=\eta(i)$ the number of its ancestors and by $\left(k_{1}(i), k_{2}(i), \ldots, k_{\eta}(i)\right)$ the sequence of indexes of the ancestors of $C^{i}$. So $W=$ $C^{k_{1}(i)} \supset C^{k_{2}(i)} \supset \ldots \supset C^{k_{\eta}(i)} \supset C^{i}$. Hence, $k_{1}(i)=1$ The cell $C^{i}$ is born at time $\underline{\kappa}\left(C^{i}\right)=\sum_{l=1}^{\eta} \tau^{*}\left(C^{k_{l}(i)}\right)$ and it dies at time $\bar{\kappa}\left(C^{i}\right)=\underline{\kappa}\left(C^{i}\right)+\tau^{*}\left(C^{i}\right)$; for $C^{1}$ this is $\underline{\kappa}\left(C^{1}\right)=0$ and $\bar{\kappa}\left(C^{1}\right)=\tau^{*}\left(C^{1}\right)$. It is useful to put $k_{\eta+1}(i)=i$.

With this notation at each time $t>0$ the tessellation $Y_{t} \wedge W$ is constituted by the cells $C^{i}$ for which $\underline{\kappa}\left(C^{i}\right) \leq t$ and $\bar{\kappa}\left(C^{i}\right)>t$. It is easy to see that at any time a.s. at most one cell dies and so a.s. at most only two cells are born.

Now we describe the generated cells as intersections of half-spaces. First note that by translation invariance follows $\Lambda([\{0\}])=0$. Hence, all the random hyperplanes $\left(d_{n, m}: n, m \in \mathbb{N}\right)$ a.s. do not contain the point 0 . Now, for a hyperplane $H \in \mathcal{H}$ such that $0 \notin H$ we denote by $H^{+}$and $H^{-}$the closed half-spaces generated by $H$ with the convention $0 \in \operatorname{int}\left(H^{+}\right)$. Hence, $C^{k_{l+1}(i)}=$ $C^{k_{l}(i)} \cap d_{k_{l}(i), z_{k_{l}(i)}}^{ \pm}$, where the sign in the upper index determines on which side of the dividing hyperplane the cell $C^{k_{l+1}(i)}$ is located. Then any cell can be represented as an intersection of $W$ with half-spaces,

$$
C^{i}=W \cap \bigcap_{l=1}^{\eta(i)} \bigcap_{m=1}^{z_{k_{l}(i)}} d_{k_{l}(i), m}^{s\left(k_{l}(i), m\right)} \cap \bigcap_{m=1}^{z_{i}-1} d_{i, m}^{s(i, m)} .
$$

In above relation we define the sign $s(j, m) \in\{+,-\}$ by the relation $C^{j} \subset$ $d_{j, m}^{s(j, m)}$, for $j, m \in \mathbb{N}$. Notice, that the origin $0 \in C^{i}$ if and only if all signs in the previous formula (2) satisfy $s\left(k_{l}(i), m\right)=+$ and $s(i, m)=+$.

Obviously the set of cells can be organized as a dyadic tree, by the relation " $C^{\prime}$ is a successor of $C$ ". This method of construction is done in [12. For the following it is important to observe that all the rejected hyperplanes $d_{k_{l}(i), m}$ are also included in this intersection because the intersection with the appropriate half-spaces does not alter the cell. Although the third set $\bigcap_{m=1}^{z_{i}-1} d_{i, m}^{s(i, m)}$ in the intersection (2) does not modify the resulting set, we also include it because we will use this representation later.

In 12 it was shown that there is no explosion, so at each time $t>0$ the number of cells of $Y_{t} \wedge W$, denoted by $\xi_{t}$, is finite a.s. Renumbering the cells, we write $\left\{C_{t}^{i}: i=1, \ldots, \xi_{t}\right\}$ for the set of cells of $Y_{t} \wedge W$.

\subsection{Independent increments relation}

The name STIT is an abbreviation for "stochastic stability under the operation of iteration of tessellations". Closely related to that stability is a certain 
independence of increments of the STIT process in time.

In order to explain the operation of iteration, for $T \in \mathbb{T}$ we number its cells in the following way. Assign to each cell a reference point in its interior (e.g. the Steiner point, see [14, p. 613, or another point that is a.s. uniquely defined). Order the set of the reference points of all cells of $T$ by its distance from the origin. For random homogeneous tessellations this order is a.s. unique. Then number the cells of $T$ according to this order, starting with number 1 for the cell which contains the origin. Thus we write $C(T)^{1}, C(T)^{2}, \ldots$ for the cells of $T$.

For $T \in \mathbb{T}$ and $\vec{R}=\left(R^{m}: m \in \mathbb{N}\right) \in \mathbb{T}^{\mathbb{N}}$, we define the tessellation $T \boxplus \vec{R}$, referred to as the iteration of $T$ and $\vec{R}$, by its set of cells

$$
T \boxplus \vec{R}=\left\{C(T)^{k} \cap C\left(R^{k}\right)^{l}: k=1, \ldots ; l=1, \ldots ; \operatorname{int}\left(C(T)^{k} \cap C\left(R^{k}\right)^{l}\right) \neq \emptyset\right\} .
$$

So, we restrict $R^{k}$ to the cell $C(T)^{k}$, and this is done for all $k=1, \ldots$ The same definition holds when the tessellation and the sequence of tessellations are restricted to some window.

To state the independence relation of the increments of the Markov process $Y$ of STIT tessellations, we fix a copy of the random process $Y$ and let $\vec{Y}^{\prime}=$ $\left(Y^{\prime m}: m \in \mathbb{N}\right)$ be a sequence of independent copies of $Y$, all of them being also independent of $Y$. In particular $Y^{\prime m} \sim Y$. For a fixed time $s>0$, we set $\vec{Y}_{s}^{\prime}=\left(Y_{s}^{\prime m}: m \in \mathbb{N}\right)$. Then, from the construction and from the consistency property of $Y$ it is straightforward to see that the following property holds

$$
Y_{t+s} \sim Y_{t} \boxplus \vec{Y}_{s}^{\prime} \text { for all } t, s>0 .
$$

This relation was firstly stated in Lemma 2 in [12]. It implies $Y_{2 t} \sim Y_{t} \boxplus \vec{Y}_{t}^{\prime}$. The STIT property means that

$$
Y_{t} \sim 2\left(Y_{t} \boxplus \vec{Y}_{t}^{\prime}\right) \text { for all } t>0,
$$

so $Y_{t} \sim 2 Y_{2 t}$. Here the multiplication with 2 stands for the transformation $x \mapsto 2 x, x \in \mathbb{R}^{\ell}$.

\section{The space of tessellations and the tail $\sigma$-algebra}

Let $\mathcal{C}$ be the set of all compact subsets of $\mathbb{R}^{\ell}$. We endow $\mathbb{T}$ with the Borel $\sigma$-algebra $\mathcal{B}(\mathbb{T})$ of the Fell topology (also known as the topology of closed convergence), namely

$$
\mathcal{B}(\mathbb{T})=\sigma(\{\{T \in \mathbb{T}: \partial T \cap C=\emptyset\}: C \in \mathcal{C}\}) .
$$

(As usual, for a class of sets $\mathcal{I}$ we denote by $\sigma(\mathcal{I})$ the smallest $\sigma$-algebra containing $\mathcal{I})$. Let us fix $\mathbf{P}$ a probability measure on $(\mathbb{T}, \mathcal{B}(\mathbb{T}))$. All the sets are determined $\bmod \mathbf{P}$, that is up to a $\mathbf{P}$-negligible set. So, for $\mathcal{E}, \mathcal{D} \in \mathcal{B}(\mathbb{T})$ we 
write $\mathcal{E}=\mathcal{D} \bmod \mathbf{P}$ if $\mathbf{P}(\mathcal{E} \Delta \mathcal{D})=0$. Also for a pair $\mathcal{B}^{\prime}, \mathcal{B}^{\prime \prime}$ of sub- $\sigma$-algebras of $\mathcal{B}(\mathbb{T})$ we write $\mathcal{B}^{\prime} \subseteq \mathcal{B}^{\prime \prime} \bmod \mathbf{P}$ if for all $\mathcal{E} \in \mathcal{B}^{\prime}$ there exists $\mathcal{D} \in \mathcal{B}^{\prime \prime}$ such that $\mathcal{E}=\mathcal{D} \bmod \mathbf{P}$.

For a window $W$ we introduce

$$
\mathcal{B}\left(\mathbb{T}_{W}\right)=\sigma(\{\{T \in \mathbb{T}: \partial T \cap C=\emptyset\}: C \subseteq W, C \in \mathcal{C}\}) .
$$

By definition $\mathcal{B}\left(\mathbb{T}_{W}\right) \subset \mathcal{B}(\mathbb{T})$ is a sub- $\sigma$-algebra. We notice that if $W^{\prime} \subseteq W$ then $\mathcal{B}\left(\mathbb{T}_{W^{\prime}}\right) \subseteq \mathcal{B}\left(\mathbb{T}_{W}\right)$.

Note that the set $\mathbb{T} \wedge W$ can be endowed with the $\sigma$-field $\mathcal{B}(\mathbb{T} \wedge W)=\sigma(\{\{T \in \mathbb{T} \wedge W: \partial T \cap C=\emptyset\}: C \subseteq W \backslash \partial W, C \in \mathcal{C}\})$. For all $\mathcal{E} \in \mathcal{B}\left(\mathbb{T}_{W}\right)$ we put $\mathcal{E} \wedge W=\{T \wedge W: T \in \mathcal{E}\}$, which belongs to $\mathcal{B}(\mathbb{T} \wedge W)$. Denoting the law of the STIT process $Y$ by $\mathbb{P}$ it holds

$$
\forall t>0, \forall \mathcal{E} \in \mathcal{B}\left(\mathbb{T}_{W}\right): \quad \mathbb{P}\left(Y_{t} \in \mathcal{E}\right)=\mathbb{P}\left(Y_{t} \wedge W \in \mathcal{E} \wedge W\right) .
$$

To avoid overburden notation and since there will be no confusion, instead of $\mathcal{E} \wedge W$ in the last formula we will only put $\mathcal{E}$, so it reads

$$
\forall t>0, \forall \mathcal{E} \in \mathcal{B}\left(\mathbb{T}_{W}\right): \quad \mathbb{P}\left(Y_{t} \in \mathcal{E}\right)=\mathbb{P}\left(Y_{t} \wedge W \in \mathcal{E}\right)
$$

Let $\left(W_{n}: n \in \mathbb{N}\right)$ be an increasing sequence of windows such that

$$
\mathbb{R}^{\ell}=\bigcup_{n \in \mathbb{N}} W_{n} \text { and } \forall n \in \mathbb{N}, W_{n} \subset \operatorname{int} W_{n+1} .
$$

We have

$$
\mathcal{B}\left(\mathbb{T}_{W_{n}}\right) \nearrow \mathcal{B}(\mathbb{T}) \text { as } n \nearrow \infty,
$$

which means $\sigma\left(\bigcup_{n \in \mathbb{N}} \mathcal{B}\left(\mathbb{T}_{W_{n}}\right)\right)=\mathcal{B}(\mathbb{T})$. On the other hand, it is easy to check that $\mathcal{B}(\mathbb{T})=\mathcal{B}(\mathbb{T})^{\mathrm{a}}$, where

$\mathcal{B}(\mathbb{T})^{\mathrm{a}}=\left\{\mathcal{E} \in \mathcal{B}(\mathbb{T}): \forall \epsilon>0, \exists n \in \mathbb{N}, \exists \mathcal{E}_{n} \in \mathcal{B}\left(\mathbb{T}_{W_{n}}\right)\right.$ such that $\left.\mathbf{P}\left(\mathcal{E} \Delta \mathcal{E}_{n}\right)<\epsilon\right\}$.

In fact by definition we have $\mathcal{B}(\mathbb{T})^{\mathrm{a}} \subseteq \mathcal{B}(\mathbb{T})$ and $\bigcup_{n \in \mathbb{N}} \mathcal{B}\left(\mathbb{T}_{W_{n}}\right) \subseteq \mathcal{B}(\mathbb{T})^{\mathrm{a}}$. Because $\mathcal{B}(\mathbb{T})^{\text {a }}$ is a $\sigma$-algebra, then necessarily $\mathcal{B}(\mathbb{T})^{\mathrm{a}}=\mathcal{B}(\mathbb{T})$.

In order to study the tail $\sigma$-algebra, we will also consider sets of tessellations which are determined by their behavior outside a window $W$, i.e. in its complement $W^{c}$. We define the $\sigma$-algebra

$$
\mathcal{B}\left(\mathbb{T}_{W^{c}}\right)=\sigma\left(\left\{\{T \in \mathbb{T}: \partial T \cap C=\emptyset\}: C \subset W^{c}, C \in \mathcal{C}\right\}\right) .
$$

We have $\mathcal{B}\left(\mathbb{T}_{W^{c}}\right) \subset \mathcal{B}(\mathbb{T})$. On the other hand, if $W^{\prime} \subseteq W$ then $\mathcal{B}\left(\mathbb{T}_{W^{c}}\right) \subseteq$ $\mathcal{B}\left(\mathbb{T}_{W^{\prime c}}\right)$.

Let $\left(W_{n}: n \in \mathbb{N}\right)$ and $\left(W_{n}^{\prime}: n \in \mathbb{N}\right)$ be a pair of increasing sequences of windows satisfying the conditions in (6). Then $\forall n, \exists m$ such that $W_{n} \subseteq W_{m}^{\prime}$ and $\forall m, \exists q$ such that $W_{m}^{\prime} \subseteq W_{q}$. This gives

$$
\mathcal{B}\left(\mathbb{T}_{W_{n}^{c}}\right) \subseteq \mathcal{B}\left(\mathbb{T}_{W_{m}^{\prime}}\right) \subseteq \mathcal{B}\left(\mathbb{T}_{W_{q}^{c}}\right)
$$


Hence $\cap_{n=1}^{\infty} \mathcal{B}\left(\mathbb{T}_{W_{n}^{c}}\right)=\cap_{n=1}^{\infty} \mathcal{B}\left(\mathbb{T}_{W_{n}^{\prime c}}\right)$. This equality allows us to define, in analogy with the definition done for point processes (see [2], Definition 12.3.IV), the tail $\sigma$-algebra on the space of tessellations.

Definition 1 The tail $\sigma$-algebra is defined as $\mathcal{B}_{-\infty}(\mathbb{T})=\bigcap_{n=1}^{\infty} \mathcal{B}\left(\mathbb{T}_{W_{n}^{c}}\right)$, where $\left(W_{n}: n \in \mathbb{N}\right)$ is an increasing sequence of windows such that for all $n \in \mathbb{N}$, $W_{n} \subset \operatorname{int} W_{n+1}$, and $\mathbb{R}^{\ell}=\bigcup_{n \in \mathbb{N}} W_{n}$.

Note that $\left(W_{n}=[-n, n]^{\ell}: n \in \mathbb{N}\right)$ satisfies (6), so it can be used in the above definition and also in the rest of the paper.

Lemma 1 Assume that for every window $W^{\prime}$, all $\mathcal{D} \in \mathcal{B}\left(\mathbb{T}_{W^{\prime}}\right)$ and all $\epsilon>0$, there exists a window $\widehat{W}$ depending on $\left(W^{\prime}, \mathcal{D}, \epsilon\right)$, such that

$$
W^{\prime} \subset \operatorname{int} \widehat{W} \quad \text { and } \forall \mathcal{E} \in \mathcal{B}\left(\mathbb{T}_{\widehat{W}^{c}}\right): \quad|\mathbf{P}(\mathcal{D} \cap \mathcal{E})-\mathbf{P}(\mathcal{D}) \mathbf{P}(\mathcal{E})|<\epsilon
$$

Then, the tail $\sigma$-algebra $\mathcal{B}_{-\infty}(\mathbb{T})$ is $\mathbf{P}$-trivial, that is

$$
\forall \mathcal{E} \in \mathcal{B}_{-\infty}(\mathbb{T}): \quad \mathbf{P}(\mathcal{E})=0 \text { or } \mathbf{P}(\mathcal{E})=1
$$

Proof 1 Let $\mathcal{D} \in \mathcal{B}_{-\infty}(\mathbb{T})$. Let $\left(W_{n}: n \in \mathbb{N}\right)$ be an increasing sequence of windows satisfying ([6). Let $\epsilon>0$ be fixed. Since $\mathcal{D} \in \mathcal{B}(\mathbb{T})$, from (7) we get,

$$
\exists k, \exists \mathcal{D}_{k} \in \mathcal{B}\left(\mathbb{T}_{W_{k}}\right) \text { such that } \mathbf{P}\left(\mathcal{D} \Delta \mathcal{D}_{k}\right)<\epsilon
$$

From hypothesis (8) there exists a window $\widehat{W}$, depending on $\left(W_{k}, \mathcal{D}_{k}, \epsilon\right)$, such that $W_{k} \subset \operatorname{int} \widehat{W}$ and $\forall \mathcal{E} \in \mathcal{B}\left(\mathbb{T}_{\widehat{W}^{c}}\right)$ it holds $\left|\mathbf{P}\left(\mathcal{D}_{k} \cap \mathcal{E}\right)-\mathbf{P}\left(\mathcal{D}_{k}\right) \mathbf{P}(\mathcal{E})\right|<\epsilon$. We know that $\exists n \geq k$ such that $\widehat{W} \subseteq W_{n}$. So, $\mathcal{B}\left(\mathbb{T}_{W_{n}^{c}}\right) \subseteq \mathcal{B}\left(\mathbb{T}_{\widehat{W}^{c}}\right)$. We then have

$$
\forall \mathcal{E} \in \mathcal{B}\left(\mathbb{T}_{W_{n}^{c}}\right): \quad\left|\mathbf{P}\left(\mathcal{D}_{k} \cap \mathcal{E}\right)-\mathbf{P}\left(\mathcal{D}_{k}\right) \mathbf{P}(\mathcal{E})\right|<\epsilon .
$$

Since $\mathcal{D} \in \mathcal{B}_{-\infty}(\mathbb{T}) \subset \mathcal{B}\left(\mathbb{T}_{W_{n}^{c}}\right)$ we get $\left|\mathbf{P}\left(\mathcal{D}_{k} \cap \mathcal{D}\right)-\mathbf{P}\left(\mathcal{D}_{k}\right) \mathbf{P}(\mathcal{D})\right|<\epsilon$. From (10) we deduce $|\mathbf{P}(\mathcal{D} \cap \mathcal{D})-\mathbf{P}(\mathcal{D}) \mathbf{P}(\mathcal{D})|<3 \epsilon$. Since this occurs for all $\epsilon>0$ we conclude $\mathbf{P}(\mathcal{D})=\mathbf{P}(\mathcal{D}) \mathbf{P}(\mathcal{D})$, so $\mathbf{P}(\mathcal{D})=1$ or 0 .

Let us discuss what happens when $\mathbf{P}$ is translation invariant. Let $h \in \mathbb{R}^{\ell}$. For any set $D \subseteq \mathbb{R}^{\ell}$ put $D+h=\{x+h: x \in D\}$ and for $T \in \mathbb{T}$ denote by $T+h$ the tessellation whose boundary is $\partial(T+h)=\partial(T)+h$. For $\mathcal{E} \subseteq \mathbb{T}$ put $\mathcal{E}+h=\{T+h: T \in \mathcal{E}\}$. For all $\mathcal{E} \in \mathcal{B}(\mathbb{T})$ we have $\mathcal{E}+h \in \mathcal{B}(\mathbb{T})$ because $\{C \in \mathcal{C}\}=\{C+h: C \in \mathcal{C}\}$. The probability measure $\mathbf{P}$ is translation invariant if it satisfies $\mathbf{P}(\mathcal{E})=\mathbf{P}(\mathcal{E}+h)$ for all $\mathcal{E} \in \mathcal{B}(\mathbb{T})$ and $h \in \mathbb{R}^{\ell}$.

A set $\mathcal{E} \in \mathcal{B}(\mathbb{T})$ is said to be $(\mathbf{P}-)$ invariant, we put $\mathcal{E} \in \mathcal{I}(\mathbb{T})$, if $\mathbf{P}(\mathcal{E} \Delta(\mathcal{E}+$ $h))=0$ for all $h \in \mathbb{R}^{\ell}$. Note that if $\mathcal{E} \in \mathcal{I}(\mathbb{T}), \mathcal{D} \in \mathcal{B}(\mathbb{T})$ and $\mathcal{E}=\mathcal{D} \bmod \mathbf{P}$, then $\mathcal{D} \in \mathcal{I}(\mathbb{T})$. It is easily checked that $\mathcal{I}(\mathbb{T}) \subseteq \mathcal{B}(\mathbb{T})$ is a sub- $\sigma$-algebra, the invariant $\sigma$-algebra (see e.g. [2]). We have the inclusion relation,

$$
\mathcal{I}(\mathbb{T}) \subseteq \mathcal{B}_{-\infty}(\mathbb{T}) \bmod \mathbf{P}
$$


We note that (11) corresponds to propositions in 2] (pp. 206-210) for random measures. For completeness we will prove (111). We denote $\mathbf{1}=(1, \ldots, 1) \in \mathbb{R}^{\ell}$, so $a \mathbf{1}=(a, \ldots, a)$ for $a \in \mathbb{R}$. Fix the sequence $\left(W_{n}=[-n, n]^{\ell}: n \in \mathbb{N}\right)$. Note that if $m>2 n$ then $W_{n}-m \mathbf{1} \subset W_{n}^{c}$.

Let $\mathcal{E} \in \mathcal{I}(\mathbb{T})$ : For all $n \in \mathbb{N}$, there exists $k=k(n)>n$ and $\mathcal{E}_{k} \in \mathcal{B}\left(\mathbb{T}_{W_{k}}\right)$ such that $\mathbf{P}\left(\mathcal{E} \Delta \mathcal{E}_{k}\right)<2^{-n}$. Since $\mathcal{E}_{k} \in \sigma\left(\left\{\{T \in \mathbb{T}: \partial T \cap C=\emptyset\}: C \subseteq W_{k}, C \in \mathcal{C}\right\}\right.$ we have that for $N>2 k$,

$$
\begin{aligned}
& \mathcal{E}_{k}-N \mathbf{1} \in \sigma\left(\left\{\{T \in \mathbb{T}: \partial T \cap C=\emptyset\}: C \subseteq W_{k}-N \mathbf{1}, C \in \mathcal{C}\right\}\right) \\
\subseteq & \sigma\left(\left\{\{T \in \mathbb{T}: \partial T \cap C=\emptyset\}: C \subset W_{k}^{c}, C \in \mathcal{C}\right\}\right)=\mathcal{B}\left(\mathbb{T}_{W_{k}^{c}}\right) .
\end{aligned}
$$

Since $\mathbf{P}(\mathcal{E} \Delta(\mathcal{E}-N \mathbf{1}))=0$ and $\mathbf{P}\left((\mathcal{E}-N \mathbf{1}) \Delta\left(\mathcal{E}_{k}-N \mathbf{1}\right)\right)=\mathbf{P}\left(\mathcal{E} \Delta \mathcal{E}_{k}\right)<2^{-n}$ we get,

$\forall N>2 k: \quad \mathbf{P}\left(\mathcal{E} \Delta\left(\mathcal{E}_{k}-N \mathbf{1}\right)\right) \leq \mathbf{P}(\mathcal{E} \Delta(\mathcal{E}-N \mathbf{1}))+\mathbf{P}\left((\mathcal{E}-N \mathbf{1}) \Delta\left(\mathcal{E}_{k}-N \mathbf{1}\right)\right)<2^{-n}$.

We have $k>1$. Take $N=k^{2}$ and use $k^{2}>2 k$ to get

$$
\mathbf{P}\left(\mathcal{E} \Delta\left(\mathcal{E}_{k}-k^{2} \mathbf{1}\right)\right) \leq 2^{-n} \text { and } \mathcal{E}_{k}-k^{2} \mathbf{1} \in \mathcal{B}\left(\mathbb{T}_{W_{k}^{c}}\right) .
$$

Define $\mathcal{D}_{m}=\bigcup_{n>m}\left(\mathcal{E}_{k(n)}-k(n)^{2} \mathbf{1}\right)$ for $m \geq 1$. This sequence of sets satisfies $\mathbf{P}\left(\mathcal{E} \Delta \mathcal{D}_{m}\right) \leq 2^{-m}, \mathcal{D}_{m} \in \mathcal{B}\left(\mathbb{T}_{W_{k(m)}^{c}}\right)$ and $\mathcal{D}_{m}$ decreases with $m$. We conclude that $\mathcal{D}=\bigcap_{m>1} \mathcal{D}_{m}$ satisfies $\mathbf{P}(\mathcal{E} \Delta \mathcal{D})=0$ and $\mathcal{D} \in \mathcal{B}_{-\infty}(\mathbb{T})$. Hence, relation (11) holds.

So, when the tail $\sigma$-algebra $\mathcal{B}_{-\infty}(\mathbb{T})$ is $\mathbf{P}$-trivial, then $\mathbf{P}$ is ergodic with respect to translations, because every invariant set $\mathcal{E} \in \mathcal{I}(\mathbb{T})$ also belongs to $\mathcal{B}_{-\infty}(\mathbb{T})$ and so $\mathbf{P}(\mathcal{E})=0$ or $\mathbf{P}(\mathcal{E})=1$. We also have that if $\mathbf{P}$ is translation invariant and $\mathcal{B}_{-\infty}(\mathbb{T})$ is $\mathbf{P}$-trivial, then the action of translations is mixing. That is,

$\mathcal{B}_{-\infty}(\mathbb{T})$ is $\mathbf{P}$ - trivial $\Rightarrow \forall \mathcal{D}, \mathcal{E} \in \mathcal{B}(\mathbb{T}): \lim _{|h| \rightarrow \infty} \mathbf{P}(\mathcal{D} \cap(\mathcal{E}+h))=\mathbf{P}(\mathcal{D}) \mathbf{P}(\mathcal{E})$

This result is shown for random measures in Proposition 12.3.V. in 2. But for completeness let us prove it. Let $\mathcal{D}, \mathcal{E} \in \mathcal{B}(\mathbb{T})$ and fix $\epsilon>0$. From (7) follows the existence of $k$ and $\mathcal{E}_{k} \in \mathcal{B}\left(\mathbb{T}_{W_{k}}\right)$ such that $\mathbf{P}\left(\mathcal{E} \Delta \mathcal{E}_{k}\right)<\epsilon$. For every $h \in \mathbb{R}^{\ell}$ we have

$$
\mid \mathbf{P}\left(\mathcal{D} \cap(\mathcal{E}+h)-\mathbf{P}\left(\mathcal{D} \cap\left(\mathcal{E}_{k}+h\right) \mid<\epsilon .\right.\right.
$$

Our choice $W_{n}=[-n, n]^{\ell}$ implies that for all $h \in \mathbb{R}^{\ell}, N \in \mathbb{N}$ with $N>k$ and $|h|>(N+k) \sqrt{\ell}$ we have $\mathcal{E}_{k}+h \in \mathcal{B}\left(\mathbb{T}_{W_{N}^{c}}\right), W_{k} \cap W_{N}^{c}=\emptyset$, and so

$$
\mathbf{P}\left(\mathcal{D} \cap\left(\mathcal{E}_{k}+h\right)\right)=\mathbb{E}\left(\mathbb{E}\left(\mathbf{1}_{\mathcal{E}_{k}+h} \mathbf{1}_{\mathcal{D}} \mid \mathcal{B}\left(\mathbb{T}_{W_{N}^{c}}\right)\right)=\mathbb{E}\left(\mathbf{1}_{\mathcal{E}_{k}+h} \mathbb{E}\left(\mathbf{1}_{\mathcal{D}} \mid \mathcal{B}\left(\mathbb{T}_{W_{N}^{c}}\right)\right) .\right.\right.
$$

The Decreasing Martingale Theorem (see e.g. [13]) yields

$$
\lim _{N \rightarrow \infty} \mathbb{E}\left(\mathbf{1}_{\mathcal{D}} \mid \mathcal{B}\left(\mathbb{T}_{W_{N}^{c}}\right)=\mathbb{E}\left(\mathbf{1}_{\mathcal{D}} \mid \mathcal{B}_{-\infty}(\mathbb{T})\right) \text { in } L^{1}(\mathbf{P})\right.
$$


Since $\mathcal{B}_{-\infty}(\mathbb{T})$ is assumed to be $\mathbf{P}$-trivial we have $\mathbb{E}\left(\mathbf{1}_{\mathcal{D}} \mid \mathcal{B}_{-\infty}(\mathbb{T})\right)=\mathbf{P}(\mathcal{D})$. So $\lim _{N \rightarrow \infty} \mathbb{E}\left(\mathbf{1}_{\mathcal{D}} \mid \mathcal{B}\left(\mathbb{T}_{W_{N}^{c}}\right)=\mathbf{P}(\mathcal{D})\right.$ in $L^{1}(\mathbf{P})$. Let $N$ be sufficiently large in order that $\| \mathbb{E}\left(\mathbf{1}_{\mathcal{D}} \mid \mathcal{B}\left(\mathbb{T}_{W_{N}^{c}}\right)-\mathbf{P}(\mathcal{D}) \|_{1}<\epsilon\right.$. Then for $|h|>(N+k) \sqrt{\ell}$ we can use (13) to obtain,

$$
\left|\mathbf{P}\left(\mathcal{D} \cap\left(\mathcal{E}_{k}+h\right)\right)-\mathbf{P}(\mathcal{D}) \mathbf{P}\left(\mathcal{E}_{k}+h\right)\right| \leq \| \mathbb{E}\left(\mathbf{1}_{\mathcal{D}} \mid \mathcal{B}\left(\mathbb{T}_{W_{N}^{c}}\right)-\mathbf{P}(\mathcal{D}) \|_{1}<\epsilon .\right.
$$

Since $\mathbf{P}\left(\mathcal{E}_{k}+h\right)=\mathbf{P}\left(\mathcal{E}_{k}\right)$ we deduce $\mathbf{P}\left(\mathcal{D} \cap\left(\mathcal{E}_{k}+h\right)\right) \rightarrow \mathbf{P}(\mathcal{D}) \mathbf{P}\left(\mathcal{E}_{k}\right)$ as $h \rightarrow \infty$. We conclude $\mathbf{P}(\mathcal{D} \cap(\mathcal{E}+h)) \rightarrow \mathbf{P}(\mathcal{D}) \mathbf{P}(\mathcal{E})$ as $h \rightarrow \infty$, so mixing is shown.

\section{Main results}

As already defined, $Y=\left(Y_{t}: t>0\right)$ denotes a STIT tessellation process, defined by the measure $\Lambda$ on $\mathcal{H}$ in $\mathbb{R}^{\ell}$ which satisfies the properties described in Section 2.1

Theorem 1 Let $W^{\prime}$ be a window. Then

$$
\begin{aligned}
& \forall t>0, \forall \varepsilon>0, \exists \text { a window } \widehat{W} \text { such that } W^{\prime} \subset \operatorname{int} \widehat{W}, \text { and } \\
& \forall \mathcal{D} \in \mathcal{B}\left(\mathbb{T}_{W^{\prime}}\right), \forall \mathcal{E} \in \mathcal{B}\left(\mathbb{T}_{\widehat{W}^{c}}\right):\left|\mathbb{P}\left(Y_{t} \in \mathcal{D} \cap \mathcal{E}\right)-\mathbb{P}\left(Y_{t} \in \mathcal{D}\right) \mathbb{P}\left(Y_{t} \in \mathcal{E}\right)\right|<\varepsilon .
\end{aligned}
$$

Denote by $\mathbf{P}^{t}$ the marginal law of $Y_{t}$ in $\mathbb{T}$, that is

$$
\forall \mathcal{E} \in \mathcal{B}(\mathbb{T}): \quad \mathbf{P}^{t}(\mathcal{E})=\mathbb{P}\left(Y_{t} \in \mathcal{E}\right)
$$

We say that at time $t>0$ the tail $\sigma$-algebra $\mathcal{B}_{-\infty}(\mathbb{T})$ is trivial for the STIT process $Y$ if $\mathbf{P}^{t}$ is trivial.

Theorem 1 implies that $\mathbf{P}^{t}$ satisfies the sufficient condition (8), which by Lemma 11implies the triviality of the tail $\sigma$-algebra $\mathcal{B}_{-\infty}(\mathbb{T})$. Hence, the following result holds.

Theorem 2 For all $t>0$ the tail $\sigma$-algebra is trivial for the STIT process $Y$, that is $\forall \mathcal{E} \in \mathcal{B}_{-\infty}(\mathbb{T})$ we have $\mathbb{P}\left(Y_{t} \in \mathcal{E}\right)=0$ or $\mathbb{P}\left(Y_{t} \in \mathcal{E}\right)=1$.

Relation (12) ensures that Theorem 2 is stronger than the mixing property shown by Lachièze-Rey $[8]$.

\section{Proof of Theorem 1}

Let $W^{\prime}$ be a window. Since the measure $\Lambda$ is supposed to be translation invariant, without loss of generality it can be assumed that the origin $0 \in \operatorname{int}\left(W^{\prime}\right)$.

Let $W$ be a window such that $W^{\prime} \subset \operatorname{int}(W)$. A key idea is the investigation of the probability that the window $W^{\prime}$ and the complement $W^{c}$ are separated by the STIT process $Y \wedge W$ (we say $W^{\prime}$ encapsulated within $W$ ) and thus the 
construction inside $W^{\prime}$ and outside $W$, respectively, are approximately independent.

For simplicity, denote by $C_{t}=C_{t}^{1}$ the (a.s. uniquely determined) cell of tessellation $Y_{t} \wedge W$ that contains the origin in its interior. Obviously, $C_{0}=W$. Note that $C_{t}$ decreases as time $t$ increases. On the other hand since $W^{\prime} \subset W$, when we consider the STIT on $W^{\prime}$ we can take $\left(Y_{t} \wedge W\right) \wedge W^{\prime}$.

Definition 2 Let be $W^{\prime}, W$ be two windows with $0 \in W^{\prime} \subset \operatorname{int}(W)$ and let $t>0$. We say that $W^{\prime}$ is encapsulated inside $W$ at time $t$ if the cell $C_{t}$ that contains 0 in $Y_{t} \wedge W$ is such that,

$$
W^{\prime} \subseteq C_{t} \subset \operatorname{int}(W) .
$$

We write $\left.W^{\prime}\right|_{t} W$ if $W^{\prime}$ is encapsulated inside $W$ at time $t$.

We denote the encapsulation time by

$$
\mathcal{S}\left(W^{\prime}, W\right)=\inf \left\{t>0:\left.W^{\prime}\right|_{t} W\right\}
$$

where as usual we put $\mathcal{S}\left(W^{\prime}, W\right)=\infty$ when $\left\{t>0:\left.W^{\prime}\right|_{t} W\right\}=\emptyset$. Encapsulation of $W^{\prime}$ inside $W$ means that $\mathcal{S}\left(W^{\prime}, W\right)<\infty$ or equivalently $W^{\prime}{ }_{t} W$ for some $t>0$. That is, where the boundaries $\partial W^{\prime}$ and $\partial W$ are completely separated by facets of the 0-cell before the smaller window $W^{\prime}$ is hit for the first time by a facet of the STIT tessellation.

We have $\left\{\mathcal{S}\left(W^{\prime}, W\right) \leq t\right\} \in \sigma\left(Y_{s}: s \leq t\right)$, so it is a stopping time. Hence the variable $\mathcal{S}\left(W^{\prime}, W\right)$ is also a stopping time for the processes $Y \wedge W$. On the other hand notice that the distribution of $\mathcal{S}\left(W^{\prime}, W\right)$ does neither depend on the method of construction of the STIT process $Y$, nor on the window $\widetilde{W}$ where the construction is performed as long as $W \subseteq \widetilde{W}$. In several proofs of the results we will assume that the starting process is $Y$, but in some others ones we will start from the STIT process $Y \wedge W$, as it occurs in Lemma 2

Note that even if in the STIT construction we have "independence after separation", it has to be considered that the tessellation outside $W$ also depends on the process until the separation time.

For two Borel sets $A, B \subset \mathbb{R}^{\ell}$ we denote by

$$
[A \mid B]=\left\{H \in \mathcal{H}:\left(A \subset \operatorname{int}\left(H^{+}\right) \wedge B \subset \operatorname{int}\left(H^{-}\right)\right) \vee\left(\left(A \subset \operatorname{int}\left(H^{-}\right) \wedge B \subset \operatorname{int}\left(H^{+}\right)\right)\right\},\right.
$$

the set of all hyperplanes that separate $A$ and $B$. This set is a Borel set in $\mathcal{H}$.

For a window $W$ (which is defined to be a convex polytope) we denote by $\left\{f_{a}^{W}: a=1, \ldots, q\right\}$ the $(\ell-1)$-dimensional facets of $W$. Let $W^{\prime}$ be another window such that $W^{\prime} \subset \operatorname{int}(W)$. We denote by

$$
G_{a}=\left[W^{\prime} \mid f_{a}^{W}\right], a=1, \ldots, q ;
$$


the set of hyperplanes that separate $W^{\prime}$ from the facet $f_{a}^{W}$ of $W$. Note that all these sets are non-empty, and they are not necessarily pairwise disjoint.

There exists a finite family $\left\{G_{a}^{\prime}: a=1, \ldots, q\right\}$ of pairwise disjoint nonempty measurable sets that satisfy

$$
\forall a \in\{1, \ldots, q\}, G_{a}^{\prime} \subseteq G_{a} .
$$

(E.g. we can choose $G_{a}^{\prime}=G_{a} \backslash \bigcup_{b<a} G_{b}$, or we can partition $\bigcup_{a=1}^{q} G_{a}$ alternatively.)

Lemma 2 Let $W^{\prime}, W$ be two compact convex polytopes, with $W^{\prime} \subset \operatorname{int}(W)$. Let $\left\{G_{a}^{\prime}: a=1, \ldots, q\right\}$ be a finite class of nonempty disjoint measurable sets satisfying (15) and such that $\Lambda\left(G_{a}^{\prime}\right)>0$ for all $a=1, \ldots, q$. Then,

$$
\begin{aligned}
\mathbb{P}\left(\mathcal{S}\left(W^{\prime}, W\right) \leq t\right) \geq & \mathrm{e}^{-t \Lambda\left(\left[W^{\prime}\right]\right)} \prod_{a=1}^{q}\left(1-\mathrm{e}^{-t \Lambda\left(G_{a}^{\prime}\right)}\right) \\
& +\int_{0}^{t} \Lambda\left(\left[W^{\prime}\right]\right) \mathrm{e}^{-x \Lambda\left(\left[W^{\prime}\right]\right)} \prod_{a=1}^{q}\left(1-\mathrm{e}^{-x \Lambda\left(G_{a}^{\prime}\right)}\right) \mathrm{d} x .
\end{aligned}
$$

Proof 2 Our starting point in the proof is the construction of the process $Y \wedge W$. Because we assume $0 \in W^{\prime}$ we focus on the genesis of the 0 -cell $C_{t}=C_{t}^{1}$, $t \geq 0$, only. We use the representation (2), and we emphasize that in this intersection also participate the positive half-spaces of those hyperplanes $d_{i, m}$ which are rejected in the construction.

Now, we consider a Poisson point process

$$
\Phi=\left\{\left(d_{m}, S_{m}\right): m \in \mathbb{N}\right\}
$$

on $[W] \times[0, \infty)$ with the intensity measure $\Lambda^{W} \otimes \Lambda([W]) \lambda_{+}$, where $\lambda_{+}$denotes the Lebesgue measure on $\mathbb{R}_{+}=[0, \infty)$. This point process can be considered as a marked hyperplane process where the marks are birth-times (or as a "rain of hyperplanes"). This choice of the intensity measure corresponds to the families D and $\tau$ of random variables in Subsection 2.1]: the interval between two sequential births of hyperplanes is exponentially distributed with parameter $\Lambda([W])$, and the law of the born hyperplanes is $\Lambda^{W}$. Thus the $S_{m}$ are sums of i.i.d. exponentially distributed random variables which are independent of the $d_{m}$. This corresponds to one of the standard methods to construct (marked) Poisson point processes (cf. e.g. [7]). Note that this Poisson process is used for the construction of the 0 -cell exclusively.

Let $\eta$ denote the number of ancestors of $C_{t}$ with indexes $k_{1}, \ldots, k_{\eta}$, and $Z_{k_{l}}=\sum_{m=1}^{l} z_{k_{l}}, l=1, \ldots, \eta+1$. Thus we can write formula (2) for the 0-cell as

$$
C_{t}=W \cap \bigcap_{l=1}^{\eta} \bigcap_{m=Z_{k_{l-1}}+1}^{Z_{k_{l}}} d_{m}^{+} \cap \bigcap_{m=Z_{k_{\eta}+1}}^{Z_{k_{\eta+1}}-1} d_{m}^{+}=W \cap \bigcap_{m: S_{m}<t} d_{m}^{+} .
$$


Define the random times

$$
\begin{aligned}
\sigma^{\prime} & =\min \left\{S: \exists(d, S) \in \Phi: d \in\left[W^{\prime}\right]\right\} \text { and } \\
\sigma_{a} & =\min \left\{S: \exists(d, S) \in \Phi: d \in G_{a}^{\prime}\right\}, a=1, \ldots q
\end{aligned}
$$

These are the first times that a hyperplane of $\Phi$ falls into the respective sets. Note that these minima exist and are greater than 0 because all $\Lambda_{[W]}\left(G_{a}^{\prime}\right)>0$, and we are working on a bounded window $W$ and $\Lambda$ is assumed to be locally finite, so $\Lambda^{W}$ is a probability measure. Let

$$
\mathcal{M}=\max \left\{\sigma_{a}: a=1, \ldots q\right\}
$$

By definition for all $a=1, \ldots, q$ there exists $a\left(d_{(a)}, S_{(a)}\right) \in \Phi$ with $d_{(a)} \in G_{a}^{\prime}$ and $S_{(a)} \leq \mathcal{M}$. Then, $f_{a}^{W} \subset d_{(a)}^{-}$and $C_{S_{(a)}} \subseteq d_{(a)}^{+}$. Since $C_{\mathcal{M}} \subseteq C_{S_{(a)}}$ we deduce

$$
C_{\mathcal{M}} \subseteq \bigcap_{a=1}^{q} d_{(a)}^{+} \subset \operatorname{int}(W) .
$$

On the other hand, if $\sigma^{\prime} \geq \mathcal{M}$ then $W^{\prime}$ is not intersected until the time $\mathcal{M}=$ $\max \left\{\sigma_{a}: a=1, \ldots, q\right\}$, so we have $W^{\prime} \subseteq C_{\mathcal{M}}$. Then

$$
W^{\prime} \subseteq C_{\mathcal{M}} \subset \operatorname{int}(W)
$$

We have shown

$$
\left\{\mathcal{M} \leq \sigma^{\prime}\right\} \subseteq\left\{\mathcal{S}\left(W^{\prime}, W\right) \leq \mathcal{M}\right\}
$$

This relation implies straightforwardly the inclusion,

$$
\left\{\mathcal{M} \leq \min \left\{\sigma^{\prime}, t\right\}\right\} \subseteq\left\{\mathcal{S}\left(W^{\prime}, W\right) \leq t\right\}
$$

Indeed, from $\mathcal{M} \leq \sigma^{\prime}$ we get $\mathcal{S}\left(W^{\prime}, W\right) \leq \mathcal{M}$, and we use $\mathcal{M} \leq t$ to get relation (19). We deduce

$$
\mathbb{P}\left(\mathcal{S}\left(W^{\prime}, W\right) \leq t\right) \geq \mathbb{P}\left(\mathcal{M} \leq \min \left\{\sigma^{\prime}, t\right\}\right)
$$

Now, the sets $\left[W^{\prime}\right], G_{a}^{\prime}, a=1, \ldots, q$, are pairwise disjoint and therefore the restricted Poisson point processes $\Phi \cap\left(\left[W^{\prime}\right] \times[0, \infty)\right), \Phi \cap\left(G_{a}^{\prime} \times[0, \infty)\right)$, $a=1, \ldots, q$, are independent. Hence $\sigma^{\prime}, \sigma_{a}, a=1, \ldots, q$, are independent random variables. Then $\sigma^{\prime}$ and $\mathcal{M}$ are independent and we get

$$
\begin{aligned}
\mathbb{P}\left(\mathcal{M} \leq \min \left\{\sigma^{\prime}, t\right\}\right) & =\mathbb{P}\left(\mathcal{M} \leq \sigma^{\prime} \leq t\right)+\mathbb{P}\left(\mathcal{M} \leq t \leq \sigma^{\prime}\right) \\
& =\mathbb{P}\left(\mathcal{M} \leq \sigma^{\prime} \leq t\right)+\mathbb{P}(\mathcal{M} \leq t) \mathbb{P}\left(t \leq \sigma^{\prime}\right) .
\end{aligned}
$$

Since $\sigma^{\prime}, \sigma_{a}, a=1, \ldots, q$, are exponentially distributed with the respective parameters $\Lambda\left(\left[W^{\prime}\right]\right), \Lambda\left(G_{a}^{\prime}\right)>0, a=1, \ldots, q$, we find

$$
\mathbb{P}\left(t \leq \sigma^{\prime}\right)=\mathrm{e}^{-t \Lambda\left(\left[W^{\prime}\right]\right)} \text { and } \mathbb{P}(\mathcal{M} \leq t)=\prod_{a=1}^{q}\left(1-\mathrm{e}^{-t \Lambda\left(G_{a}^{\prime}\right)}\right)
$$


Now, by denoting the density functions of $\sigma^{\prime}$ by $p^{\prime}$ and those ones of $\sigma_{i}$ by $p_{i}$ respectively, we get

$$
\begin{aligned}
\mathbb{P}\left(\mathcal{M} \leq \sigma^{\prime} \leq t\right) & =\int_{0}^{t} p^{\prime}(x)\left(\int_{0}^{x} p_{1}\left(x_{1}\right) \mathrm{d} x_{1} \ldots \int_{0}^{x} p_{q}\left(x_{q}\right) \mathrm{d} x_{q}\right) \mathrm{d} x \\
& =\int_{0}^{t} \Lambda\left(\left[W^{\prime}\right]\right) \mathrm{e}^{-x \Lambda\left(\left[W^{\prime}\right]\right)} \prod_{a=1}^{q}\left(1-\mathrm{e}^{-x \Lambda\left(G_{a}^{\prime}\right)}\right) \mathrm{d} x .
\end{aligned}
$$

Therefore, formula (16) follows.

Remark 1 (i) We emphasize that $\mathcal{M} \leq \min \left\{\sigma^{\prime}, t\right\}$ is sufficient but not necessary for $\mathcal{S}\left(W^{\prime}, W\right) \leq t$. There can also be other ways to encapsulate $W^{\prime}$ within $W$ than separating the complete facets of $W$ by single hyperplanes. Alternative geometric constructions are possible.

(ii) It is well known in convex geometry, that $\left[W^{\prime} \mid f_{a}^{W}\right] \neq \emptyset$ for all $a=1, \ldots, q$. But depending on the support of the measure $\Lambda$ (in particular, if $\Lambda$ is concentrated on a set of hyperplanes with only finitely many directions) there can be windows $W$ such that $\Lambda\left(\left[W^{\prime} \mid f_{a}^{W}\right]\right)=0$ for some $a$. In such cases the bound given in Lemma回 is useless. Therefore, in the following, $W$ will be adapted to $\Lambda$ in order to have all $\Lambda\left(G_{a}^{\prime}\right)>0$. But here we will not try to find an optimal $W$ in the sense that the quantities $\Lambda\left(G_{a}^{\prime}\right)$ could be somehow maximized.

(iii) As an example consider the particular measure $\Lambda_{\perp}=\sum_{c=1}^{\ell} g_{c} \delta_{c}$, with $g_{c}>0, \delta_{c}$ the translation invariant measure on the set of all hyperplanes that are orthogonal to the $c$-th coordinate axis in $\mathbb{R}^{\ell}$, with the normalization $\delta_{c}\left(\left[s_{c}\right]\right)=1$, where $s_{c}$ is a linear segment of length 1 and parallel to the $c$-th coordinate axis. Let $W^{\prime}=[-\alpha, \alpha]^{\ell}, W=[-\beta, \beta]^{\ell}$ be two windows with $0<\alpha<\beta$. Then we can choose the sets $G_{a}^{\prime}=G_{a}$ if the facet $f_{a}^{W}$ of $W$ is orthogonal to the c-th coordinate axis, $a=1, \ldots, 2 \ell$. We have $\Lambda_{\perp}\left(G_{a}^{\prime}\right)=g_{c}(\beta-\alpha)$. Simple geometric considerations yield,

$$
\left\{\mathcal{M} \leq \min \left\{\sigma^{\prime}, t\right\}\right\}=\left\{\mathcal{S}\left(W^{\prime}, W\right) \leq t\right\} \quad \text { a.s. }
$$

and hence for $\Lambda_{\perp}$ we have the equality sign in (16).

We will use the following parameterization of hyperplanes. Let $\mathbb{S}^{\ell-1}$ be the unit hypersphere in $\mathbb{R}^{\ell}$. For $H \in \mathcal{H}, d(h) \in \mathbb{R}$ denotes its signed distance from the origin and $u(h) \in \mathbb{S}^{\ell-1}$ is its normal direction. We denote by $H(u, d)$ the hyperplane with the respective parameters $(u, d) \in \mathbb{R} \times \mathbb{S}^{\ell-1}$. The image of the non-zero, locally finite and translation invariant measure $\Lambda$ with respect to this parameterization can be written as the product measure

$$
\gamma \cdot \lambda \otimes \theta,
$$

where $\gamma>0$ is a constant, $\lambda$ is the Lebesgue measure on $\mathbb{R}$ and $\theta$ is an even probability measure on $\mathbb{S}^{\ell-1}$ (cf, e.g. [14], Theorem 4.4.1 and Theorem 13.2.12). 
Here $\theta$ is even means $\theta(A)=\theta(-A)$ for all Borel sets $A \subseteq \mathbb{S}^{\ell-1}$. The property that there is no line in $\mathbb{R}^{\ell}$ such that all hyperplanes of the support of $\Lambda$ are parallel to it, is equivalent to the property that $\theta$ is not concentrated on a great subsphere of $\mathbb{S}^{\ell-1}$, i.e there is no one-dimensional subspace $L_{1}$ of $\mathbb{R}^{\ell}$ (with the orthogonal complement $L_{1}^{\perp}$ ) such that the support of $\theta$ equals $\mathbb{G}=L_{1}^{\perp} \cap \mathbb{S}^{\ell-1}$.

Recall that $W^{\prime}$ is a window with $0 \in \operatorname{int}\left(W^{\prime}\right)$.

Lemma 3 There exists a compact convex polytope $W$ with facets $f_{1}^{W}, \ldots, f_{2 \ell}^{W}$ and $W^{\prime} \subset \operatorname{int}(W)$, and pairwise disjoint sets $G_{a}^{\prime} \subseteq\left[W^{\prime} \mid f_{a}^{W}\right]$ such that $\Lambda\left(G_{a}^{\prime}\right)>0$ for all $a=1, \ldots, 2 \ell$.

Proof 3 For $u \in \mathbb{S}^{\ell-1}$ we denote by $H_{W^{\prime}}(u)$ the supporting (i.e. tangential) hyperplane to $W^{\prime}$ with normal direction $u \in \mathbb{S}^{\ell-1}$. By $h_{W^{\prime}}(u)$ we denote the distance from the origin to $H_{W^{\prime}}(u)$. This is the support function of $W^{\prime}$, $h_{W^{\prime}}(u)=\max \left\{\langle x, u\rangle: x \in W^{\prime}\right\}$ (see [14], p. 600). With this notation we have $H_{W^{\prime}}(u)=H\left(u, h_{W^{\prime}}(u)\right)$. Note that for $d \in \mathbb{R}$ the hyperplane $H(u, d)$ is parallel to $H_{W^{\prime}}(u)$ at signed distance $d$ from the origin.

The shape of the window $W$ will depend on $\Lambda$. We use some ideas of the proof of Theorem 10.3.2 in [14]. Under the given assumptions on the support of $\Lambda$ there exist points $u_{1}, \ldots, u_{2 \ell} \in \mathbb{S}^{\ell-1}$ which all belong to the support of $\theta$ and $0 \in \operatorname{int}\left(\operatorname{conv}\left\{u_{1}, \ldots, u_{2 \ell}\right\}\right)$, i.e. the origin is in the interior of the convex hull. Now, the facets $f_{a}^{W}$ of $W$ are chosen to have normals $u_{a}$, and their distance from the origin is $h_{W^{\prime}}\left(u_{a}\right)+3, a=1, \ldots, 2 \ell$. Formally,

$$
W=\bigcap_{a=1}^{2 \ell} H\left(u_{a}, h_{W^{\prime}}\left(u_{a}\right)+3\right)^{+} .
$$

Notice that the described condition on the choice of the directions $u_{1}, \ldots, u_{2 \ell}$ guarantees that $W$ is bounded (see the proof of Theorem 10.3.2 in [14]).

The definition of the support of a measure and some continuity arguments (applied to sets of hyperplanes) yield that for all $u_{a}$ there are pairwise disjoint neighborhoods $U_{a} \subset \mathbb{S}^{\ell-1}$ such that $\theta\left(U_{a}\right)>0$, and for the sets of hyperplanes

$$
G_{a}^{\prime}=\left\{H \in \mathcal{H}: u(H) \in U_{a}, h_{W^{\prime}}\left(u_{a}\right)+1<d(H)<h_{W^{\prime}}\left(u_{a}\right)+2\right\},
$$

it holds $G_{a}^{\prime} \subset\left[W^{\prime} \mid f_{a}^{W}\right]$. Hence $\Lambda\left(\left[W^{\prime} \mid f_{a}^{W}\right]\right) \geq \Lambda\left(G_{a}^{\prime}\right)=\gamma \theta\left(U_{a}\right)>0$ for all $a=1, \ldots, 2 \ell$. Since the $U_{a}$ are pairwise disjoint, also the sets $G_{a}^{\prime}, a=1, \ldots, 2 \ell$, have this property.

Remark 2 Because the directional distribution $\theta$ is assumed to be an even measure, in the construction above one can choose $u_{\ell+a}=-u_{a}, a=1, \ldots, \ell$. Then the facets $f_{a}^{W}$ and $f_{\ell+a}^{W}$ are parallel. 
Lemma 4 The compact convex polytope $W$ constructed in Lemma 3 also satisfies the following property: $\forall \varepsilon>0, \exists t^{*}(\varepsilon)>0$ such that the following encapsulation time relation holds,

$$
\forall s \in\left(0, t^{*}(\varepsilon)\right], \exists r(s) \geq 1 \text { such that } \forall r \geq r(s): \mathbb{P}\left(\mathcal{S}\left(W^{\prime}, r W\right) \leq s\right)>1-\varepsilon .
$$

Proof 4 Let us use the notation introduced in the Lemma 3 and in its proof. For $r>0$ we set $r G_{a}^{\prime}=\left\{r h: h \in G_{a}^{\prime}\right\}$. Then, elementary linear algebra yields that $G_{a}^{\prime} \subset\left[W^{\prime} \mid f_{a}^{W}\right]$ implies $r G_{a}^{\prime} \subset\left[W^{\prime} \mid r f_{a}^{W}\right]$ for all $r>1$. Furthermore, from (22) we find

$$
\forall a=1, \ldots, 2 \ell: \quad \Lambda\left(\left[W^{\prime} \mid r f_{a}^{W}\right]\right) \geq \Lambda\left(r G_{a}^{\prime}\right)=\gamma r \theta\left(U_{a}\right) .
$$

Now denote by

$$
L=\min \left\{\Lambda\left(G_{a}^{\prime}\right)=\gamma \theta\left(U_{a}\right): a=1, \ldots, 2 \ell\right\} .
$$

We have $L>0$, and (16) yields

$$
\begin{aligned}
& \mathbb{P}\left(\mathcal{S}\left(W^{\prime}, r W\right) \leq s\right) \\
& \geq \mathrm{e}^{-s \Lambda\left(\left[W^{\prime}\right]\right)}\left(1-\mathrm{e}^{-s r L}\right)^{2 \ell}+\int_{0}^{s} \Lambda\left(\left[W^{\prime}\right]\right) \mathrm{e}^{-x \Lambda\left(\left[W^{\prime}\right]\right)}\left(1-\mathrm{e}^{-x r L}\right)^{2 \ell} \mathrm{d} x \\
& >\mathrm{e}^{-s \Lambda\left(\left[W^{\prime}\right]\right)}\left(1-\mathrm{e}^{-s r L}\right)^{2 \ell} .
\end{aligned}
$$

Note that

$$
\forall \varepsilon \in(0,1), \exists t^{*}(\varepsilon)>0 \text { such that } \mathrm{e}^{-t^{*}(\varepsilon) \Lambda\left(\left[W^{\prime}\right]\right)}>\sqrt{1-\varepsilon} .
$$

Then for all $s \in\left(0, t^{*}(\varepsilon)\right]$ we have $\mathrm{e}^{-s \Lambda\left(\left[W^{\prime}\right]\right)}>\sqrt{1-\varepsilon}$. Furthermore, for any such $s \in\left(0, t^{*}(\varepsilon)\right]$ there is an $r(s) \geq 1$ with $\left(1-\mathrm{e}^{-s r(s) L}\right)^{2 \ell}>\sqrt{1-\varepsilon}$. This finishes the proof.

Lemma 5 For all $W^{\prime}$, all $t>0$ and all $\varepsilon>0$ and $t^{*}(\varepsilon)$ such that (23) holds, there exists $t_{1}=t_{1}(\varepsilon, t) \in\left(0, \min \left\{t, t^{*}(\varepsilon)\right\}\right)$ such that for all $t_{2} \in\left(0, t_{1}\right]$,

$$
\mathbb{P}\left(Y \wedge W^{\prime} \text { has no jump in }\left[t-t_{2}, t\right)\right)>1-\varepsilon .
$$

Proof 5 Let $\left\{C_{t}^{\prime i}: i=1, \ldots, \xi_{t}^{\prime}\right\}$ be the family of cells of the pure jump Markov process $Y_{t} \wedge W^{\prime}$. The lifetimes of $C_{t}^{\prime i}$ are exponentially distributed with the parameters $\Lambda\left(\left[C_{t}^{\prime}{ }^{\prime}\right]\right)$ and they are conditionally independent conditioned that a certain set of cells is given at time $t$. Then, given a certain set of cells at $t$, the minimum of the lifetimes is exponentially distributed with parameter $\zeta_{t}=$ $\sum_{i=1}^{\xi_{t}^{\prime}} \Lambda\left(\left[C_{t}^{\prime} i\right]\right)$. 
Notice that $\zeta_{t}$ is monotonically increasing in $t$, because if at some time a cell $C^{\prime}$ is divided into the cells $C^{\prime \prime}, C^{\prime \prime \prime}$ we have $C^{\prime}=C^{\prime \prime} \cup C^{\prime \prime \prime}$ and $\left[C^{\prime}\right]=\left[C^{\prime \prime}\right] \cup\left[C^{\prime \prime \prime}\right]$. Then, by subadditivity of $\Lambda$,

$$
\Lambda\left(\left[C^{\prime}\right]\right)=\Lambda\left(\left[C^{\prime \prime}\right] \cup\left[C^{\prime \prime \prime}\right]\right) \leq \Lambda\left(\left[C^{\prime \prime}\right]\right)+\Lambda\left(\left[C^{\prime \prime \prime}\right]\right) .
$$

Since the process $Y \wedge W^{\prime}$ has no explosion, for any fixed $t>0 \quad \exists x_{0}>0$ such that for all $s \in[0, t]$ we have $\mathbb{P}\left(\zeta_{s} \leq x_{0}\right)>\sqrt{1-\varepsilon}$. We fix $t_{1}=t_{1}(\varepsilon, t) \in$ $\left(0, \min \left\{t, t^{*}(\varepsilon)\right\}\right)$ as a value which also satisfies $\mathrm{e}^{-t_{1}(\varepsilon) x_{0}}>\sqrt{1-\varepsilon}$. This yields for all $t_{2} \in\left(0, t_{1}\right]$ that

$$
\begin{aligned}
& \mathbb{P}\left(Y \wedge W^{\prime} \text { has no jump in }\left[t-t_{2}, t\right)\right) \\
& \geq \mathbb{P}\left(Y \wedge W^{\prime} \text { has no jump in }\left[t-t_{1}, t\right)\right) \\
& =\int_{0}^{\infty} \mathbb{P}\left(Y \wedge W^{\prime} \text { has no jump in }\left[t-t_{1}, t\right) \mid \zeta_{t-t_{1}}=x\right) \mathbb{P}\left(\zeta_{t-t_{1}} \in d x\right) \\
& \geq \int_{0}^{x_{0}} \mathbb{P}\left(Y \wedge W^{\prime} \text { has no jump in }\left[t-t_{1}, t\right) \mid \zeta_{t-t_{1}}=x\right) \mathbb{P}\left(\zeta_{t-t_{1}} \in d x\right) \\
& =\int_{0}^{x_{0}} \mathrm{e}^{-t_{1} x} \mathbb{P}\left(\zeta_{t-t_{1}} \in d x\right) \geq \mathrm{e}^{-t_{1} x_{0}} \mathbb{P}\left(\zeta_{t-t_{1}} \leq x_{0}\right)>1-\varepsilon
\end{aligned}
$$

In the sequel for $t>0$ and $\varepsilon>0$ the quantity $t_{1}=t_{1}(\varepsilon, t)$ is the one given by Lemma 5

Recall that under the identification $\mathcal{E}$ with $\mathcal{E} \wedge W$ we can write $\mathbb{P}(Y \in \mathcal{E})=$ $\mathbb{P}\left(Y \wedge W^{\prime} \in \mathcal{E}\right)$ for all $\mathcal{E} \in \mathcal{B}\left(\mathbb{T}_{W^{\prime}}\right)$, see (5). This identification also allows us to put for all $\mathcal{E} \in \mathcal{B}\left(\mathbb{T}_{W^{\prime}}\right), \mathcal{D} \in \mathcal{B}\left(\mathbb{T}_{W^{c}}\right), s>0$ :

$$
\mathbb{P}\left(Y_{t} \in \mathcal{E} \cap \mathcal{D}, \mathcal{S}\left(W^{\prime}, W\right)<s\right)=\mathbb{P}\left(Y_{t} \wedge W^{\prime} \in \mathcal{E}, Y_{t} \in \mathcal{D}, \mathcal{S}\left(W^{\prime}, W\right)<s\right) .
$$

Lemma 6 For all $t>0, \varepsilon>0$ and $t_{2} \in\left(0, t_{1}\right]$, we have

$$
\forall s \in\left(0, t_{2}\right], \forall \mathcal{E} \in \mathcal{B}\left(\mathbb{T}_{W^{\prime}}\right):\left|\mathbb{P}\left(Y_{t} \in \mathcal{E}\right)-\mathbb{P}\left(Y_{t-s} \in \mathcal{E}\right)\right|<\varepsilon .
$$

Proof 6 We have

$\forall s \in\left(0, t_{2}\right]:\left\{Y \wedge W^{\prime}\right.$ has no jump in $\left.\left[t-t_{2}, t\right)\right\} \subseteq\left\{Y \wedge W^{\prime}\right.$ has no jump in $\left.[t-s, t)\right\}$.

Then,

$$
\begin{aligned}
& \left\{Y_{t} \wedge W^{\prime} \in \mathcal{E}, Y \wedge W^{\prime} \text { has no jump in }\left[t-t_{2}, t\right)\right\} \\
& =\left\{Y_{t-s} \wedge W^{\prime} \in \mathcal{E}, Y \wedge W^{\prime} \text { has no jump in }\left[t-t_{2}, t\right)\right\} .
\end{aligned}
$$

Therefore $\left\{Y_{t} \wedge W^{\prime} \in \mathcal{E}\right\} \Delta\left\{Y_{t-s} \wedge W^{\prime} \in \mathcal{E}\right\} \subseteq\left\{Y \wedge W^{\prime}\right.$ has some jump in $[t-$ $\left.\left.t_{2}, t\right)\right\}$. By using the relation $|\mathbb{P}(\Gamma)-\mathbb{P}(\Theta)| \leq \mathbb{P}(\Gamma \Delta \Theta)$, the result follows. 
In the following results $W$ is a window such that $W^{\prime} \subset \operatorname{int}(W)$. We use the notation $\mathcal{S}=\mathcal{S}\left(W^{\prime}, W\right)$ for the encapsulation time.

Proposition 1 For all $t>0, \varepsilon>0$ and $t_{2} \in\left(0, t_{1}\right]$, we have

$$
\forall \mathcal{E} \in \mathcal{B}\left(\mathbb{T}_{W^{\prime}}\right):\left|\mathbb{P}\left(Y_{t} \in \mathcal{E}\right)-\mathbb{P}\left(Y_{t} \in \mathcal{E} \mid \mathcal{S}<t_{2}\right)\right|<\varepsilon
$$

Proof 7 Let us first show,

$$
\forall s \in(0, t), \forall \mathcal{E} \in \mathcal{B}\left(\mathbb{T}_{W^{\prime}}\right): \mathbb{P}\left(Y_{t} \in \mathcal{E} \mid \mathcal{S}=s\right)=\mathbb{P}\left(Y_{t-s} \in \mathcal{E}\right)
$$

Let $\vec{Y}^{\prime}=\left(Y^{\prime m}: m \in \mathbb{N}\right)$ be a sequence of independent copies of $Y$, and also independent of $Y$. Relation (3) yields

$$
Y_{t} \wedge W^{\prime} \sim\left(Y_{s} \boxplus \vec{Y}_{t-s}^{\prime}\right) \wedge W^{\prime} \sim\left(Y_{s} \wedge W^{\prime}\right) \boxplus \vec{Y}_{t-s}^{\prime} .
$$

On the event $\mathcal{S}=s$ we have $W^{\prime} \subseteq C_{s}^{1}$, this last is the cell containing the origin at time $s$, and thus $Y_{s} \wedge W^{\prime}=\left\{W^{\prime}\right\}$. Hence, on $\mathcal{S}=s$ we have $\left(Y_{s} \wedge W^{\prime}\right) \boxplus \vec{Y}_{t-s}^{\prime} \sim$ $Y_{t-s}^{\prime 1} \wedge W^{\prime}$. Therefore,

$$
\mathbb{P}\left(Y_{t} \wedge W^{\prime} \in \mathcal{E} \mid \mathcal{S}=s\right)=\mathbb{P}\left(Y_{t-s}^{\prime 1} \wedge W^{\prime} \in \mathcal{E} \mid \mathcal{S}=s\right)=\mathbb{P}\left(Y_{t-s}^{\prime 1} \wedge W^{\prime} \in \mathcal{E}\right) .
$$

Since $Y_{t-s}^{\prime 1} \sim Y_{t-s}$, relation (25) is satisfied. Hence,

$$
\begin{aligned}
& \left|\mathbb{P}\left(Y_{t} \in \mathcal{E}\right)-\mathbb{P}\left(Y_{t} \in \mathcal{E} \mid \mathcal{S}<t_{2}\right)\right| \\
= & \left|\mathbb{P}\left(Y_{t} \in \mathcal{E}\right)-\frac{1}{\mathbb{P}\left(\mathcal{S}<t_{2}\right)} \int_{0}^{t_{2}} \mathbb{P}\left(Y_{t} \in \mathcal{E} \mid S=s\right) \mathbb{P}(\mathcal{S} \in d s)\right| \\
= & \left|\mathbb{P}\left(Y_{t} \in \mathcal{E}\right)-\frac{1}{\mathbb{P}\left(\mathcal{S}<t_{2}\right)} \int_{0}^{t_{2}} \mathbb{P}\left(Y_{t-s} \in \mathcal{E}\right) \mathbb{P}(\mathcal{S} \in d s)\right| \\
= & \frac{1}{\mathbb{P}\left(S<t_{2}\right)}\left|\int_{0}^{t_{2}} \mathbb{P}\left(Y_{t} \in \mathcal{E}\right)-\mathbb{P}\left(Y_{t-s} \in \mathcal{E}\right) \mathbb{P}(\mathcal{S} \in d s)\right| \\
\leq & \frac{1}{\mathbb{P}\left(\mathcal{S}<t_{2}\right)} \int_{0}^{t_{2}}\left|\mathbb{P}\left(Y_{t} \in \mathcal{E}\right)-\mathbb{P}\left(Y_{t-s} \in \mathcal{E}\right)\right| \mathbb{P}(\mathcal{S} \in d s)<\varepsilon,
\end{aligned}
$$

where in the last inequality we use Lemma 6 .

Lemma 6. Proposition 1 and relation (25) obviously imply the following result.

Corollary 1 For all $t>0, \varepsilon>0, t_{2} \in\left(0, t_{1}\right]$ and all $s \in\left(0, t_{2}\right)$ it holds

$$
\begin{aligned}
\forall \mathcal{E} \in \mathcal{B}\left(T_{W^{\prime}}\right): \quad & \left|\mathbb{P}\left(Y_{t-s} \in \mathcal{E}\right)-\mathbb{P}\left(Y_{t} \in \mathcal{E} \mid \mathcal{S}<t_{2}\right)\right| \\
& =\left|\mathbb{P}\left(Y_{t} \in \mathcal{E} \mid \mathcal{S}=s\right)-\mathbb{P}\left(Y_{t} \in \mathcal{E} \mid \mathcal{S}<t_{2}\right)\right|<2 \varepsilon
\end{aligned}
$$


Lemma 7 For all $t>0, \varepsilon>0$ and $t_{2} \in\left(0, t_{1}\right]$ we have for all $\mathcal{D} \in \mathcal{B}\left(\mathbb{T}_{W^{\prime}}\right), \mathcal{E} \in$ $\mathcal{B}\left(\mathbb{T}_{W^{c}}\right)$,

$$
\left|\mathbb{P}\left(Y_{t} \in \mathcal{D} \cap \mathcal{E} \mid \mathcal{S}<t_{2}\right)-\mathbb{P}\left(Y_{t} \in \mathcal{D} \mid \mathcal{S}<t_{2}\right) \mathbb{P}\left(Y_{t} \in \mathcal{E} \mid \mathcal{S}<t_{2}\right)\right|<2 \varepsilon .
$$

Proof 8 Firstly, we show the following conditional independence property,

$$
\begin{aligned}
& \forall \mathcal{D} \in \mathcal{B}\left(\mathbb{T}_{W^{\prime}}\right), \mathcal{E} \in \mathcal{B}\left(\mathbb{T}_{W^{c}}\right): \\
& \mathbb{P}\left(Y_{t} \in \mathcal{D} \cap \mathcal{E} \mid \mathcal{S}=s\right)=\mathbb{P}\left(Y_{t} \in \mathcal{D} \mid \mathcal{S}=s\right) \mathbb{P}\left(Y_{t} \in \mathcal{E} \mid \mathcal{S}=s\right) .
\end{aligned}
$$

We use the notation introduced in the proof of Proposition 1 and we also shortly write $\mathcal{E}$ instead of $\mathcal{E} \wedge W^{c}$. Also the arguments are close to the ones in the proof of relation (25).

Recall that on the event $\mathcal{S}=s$ we have $W^{\prime} \subseteq C_{s}^{1} . B y Y_{s} \boxplus\left(Y_{t-s}^{\prime m}: m \geq 2\right)$ we mean that the tessellations $Y_{t-s}^{\prime m}$ are nested only into the cells $C_{s}^{m}$ of $Y_{s}$ with $m \geq 2$, and not into the cell $C_{s}^{1}$. From (25), (26) and the independence of the random variables $Y_{s}, Y_{t-s}^{\prime m}, m \geq 1$ we obtain,

$$
\begin{aligned}
& \mathbb{P}\left(Y_{t} \wedge W^{\prime} \in \mathcal{D}, Y_{t} \in \mathcal{E} \mid \mathcal{S}=s\right) \\
= & \mathbb{P}\left(\left(Y_{s} \boxplus \vec{Y}_{t-s}^{\prime}\right) \wedge W^{\prime} \in \mathcal{D},\left(Y_{s} \boxplus \vec{Y}_{t-s}^{\prime}\right) \in \mathcal{E} \mid \mathcal{S}=s\right) \\
= & \mathbb{P}\left(Y_{t-s}^{\prime 1} \wedge W^{\prime} \in \mathcal{D},\left(Y_{s} \boxplus\left(Y_{t-s}^{\prime m}: m \geq 2\right)\right) \in \mathcal{E} \mid \mathcal{S}=s\right) \\
= & \left.\mathbb{P}\left(Y_{t-s}^{\prime 1} \wedge W^{\prime} \in \mathcal{D}\right) \mathbb{P}\left(Y_{s} \boxplus\left(Y_{t-s}^{\prime m}: m \geq 2\right)\right) \in \mathcal{E} \mid \mathcal{S}=s\right) \\
= & \mathbb{P}\left(Y_{t} \wedge W^{\prime} \in \mathcal{D} \mid \mathcal{S}=s\right) \mathbb{P}\left(Y_{t} \in \mathcal{E} \mid \mathcal{S}=s\right) .
\end{aligned}
$$

Then (27) is verified. By using this equality and Corollary 1 we find,

$$
\begin{aligned}
& \mathbb{P}\left(Y_{t} \in \mathcal{D} \mid \mathcal{S}<t_{2}\right) \mathbb{P}\left(Y_{t} \in \mathcal{E} \mid \mathcal{S}<t_{2}\right)-2 \varepsilon \\
\leq & \left(\mathbb{P}\left(Y_{t} \in \mathcal{D} \mid \mathcal{S}<t_{2}\right)-2 \varepsilon\right) \mathbb{P}\left(Y_{t} \in \mathcal{E} \mid \mathcal{S}<t_{2}\right) \\
= & \frac{1}{\mathbb{P}\left(S<t_{2}\right)} \int_{0}^{t_{2}}\left(\mathbb{P}\left(Y_{t} \in \mathcal{D} \mid \mathcal{S}<t_{2}\right)-2 \varepsilon\right) \mathbb{P}\left(Y_{t} \in \mathcal{E} \mid \mathcal{S}=s\right) \mathbb{P}(\mathcal{S} \in d s) \\
< & \frac{1}{\mathbb{P}\left(\mathcal{S}<t_{2}\right)} \int_{0}^{t_{2}} \mathbb{P}\left(Y_{t} \in \mathcal{D} \mid \mathcal{S}=s\right) \mathbb{P}\left(Y_{t} \in \mathcal{E} \mid \mathcal{S}=s\right) \mathbb{P}(\mathcal{S} \in d s) \\
= & \frac{1}{\mathbb{P}\left(\mathcal{S}<t_{2}\right)} \int_{0}^{t_{2}} \mathbb{P}\left(Y_{t} \in \mathcal{D} \cap \mathcal{E} \mid \mathcal{S}=s\right) \mathbb{P}(\mathcal{S} \in d s) \\
= & \mathbb{P}\left(Y_{t} \in \mathcal{D} \cap \mathcal{E} \mid \mathcal{S}<t_{2}\right) .
\end{aligned}
$$

In an analogous way it is proven,

$$
\mathbb{P}\left(Y_{t} \in \mathcal{D} \cap \mathcal{E} \mid \mathcal{S}<t_{2}\right)<\mathbb{P}\left(Y_{t} \in \mathcal{D} \mid \mathcal{S}<t_{2}\right) \mathbb{P}\left(Y_{t} \in \mathcal{E} \mid \mathcal{S}<t_{2}\right)+2 \varepsilon,
$$

which finishes the proof.

We summarize. The window $W^{\prime}$ was fixed and there was no loss of generality in assuming $0 \in \operatorname{int}\left(W^{\prime}\right)$. Let $t>0$ and $\varepsilon>0$ be fixed. We construct $t_{1}=$ 
$t_{1}(\varepsilon, t) \in\left(0, \min \left\{t, t^{*}(\varepsilon)\right\}\right)$. Now let $W$ be as in Lemma 3. Note that for all $t_{2} \in\left(0, t_{1}(\varepsilon)\right]$ we have $\mathrm{e}^{-t_{2} \Lambda\left(\left[W^{\prime}\right]\right)}>\sqrt{1-\varepsilon}$. Then, from Lemma 4 we get that for all $t_{2} \in\left(0, t_{1}(\varepsilon)\right]$ there exists $r\left(t_{2}\right) \geq 1$ such that $\mathbb{P}\left(\mathcal{S}\left(W^{\prime}, r \widehat{W}\right)<t_{2}\right)>1-\varepsilon$ for all $r \geq r\left(t_{2}\right)$.

Now we fix $t_{2} \in\left(0, t_{1}(\varepsilon)\right]$ and define $\widehat{W}=r\left(t_{2}\right) W$. We have $W^{\prime} \subset \operatorname{int}(\widehat{W})$. From Lemma 7 we deduce the following result.

Corollary 2 For all $\mathcal{D} \in \mathcal{B}\left(\mathbb{T}_{W^{\prime}}\right), \mathcal{E} \in \mathcal{B}\left(\mathbb{T}_{\widehat{W}^{c}}\right)$ it is satisfied

$$
\left|\mathbb{P}\left(Y_{t} \in \mathcal{D} \cap \mathcal{E} \mid \mathcal{S}<t_{2}\right)-\mathbb{P}\left(Y_{t} \in \mathcal{D} \mid \mathcal{S}<t_{2}\right) \mathbb{P}\left(Y_{t} \in \mathcal{E} \mid \mathcal{S}<t_{2}\right)\right|<2 \varepsilon
$$

Proposition 2 For the window $W^{\prime}$, for all $t>0$ and $\varepsilon>0$, the window $\widehat{W}$ satisfies,

$$
\forall \mathcal{D} \in \mathcal{B}\left(\mathbb{T}_{W^{\prime}}\right), \mathcal{E} \in \mathcal{B}\left(\mathbb{T}_{\widehat{W}^{c}}\right):\left|\mathbb{P}\left(Y_{t} \in \mathcal{D} \cap \mathcal{E}\right)-\mathbb{P}\left(Y_{t} \in \mathcal{D}\right) \mathbb{P}\left(Y_{t} \in \mathcal{E}\right)\right|<4 \varepsilon
$$

Proof 9 Denote

$$
\Gamma=\left\{Y_{t} \in \mathcal{D}\right\}, \Theta=\left\{Y_{t} \in \mathcal{E}\right\}, \Upsilon=\left\{\mathcal{S}\left(W^{\prime}, \widehat{W}\right)<t_{2}\right\}
$$

We have

$$
|\mathbb{P}(\Gamma \cap \Theta \mid \Upsilon)-\mathbb{P}(\Gamma \mid \Upsilon) \mathbb{P}(\Theta \mid \Upsilon)|<2 \varepsilon \text { and } \mathbb{P}(\Upsilon)>1-\varepsilon
$$

Observe that $\mathbb{P}(\Upsilon)>1-\varepsilon$ implies, $\mathbb{P}(\Xi)-\mathbb{P}(\Xi \cap \Upsilon)<\varepsilon$ and $\mathbb{P}(\Xi)-\mathbb{P}(\Xi \cap$ $\Upsilon) \mathbb{P}(\Upsilon)<2 \varepsilon$ for all events $\Xi$, in particular when $\Xi$ is $\Gamma, \Theta$ or $\Gamma \cap \Theta$.

The first relation in (28) obviously implies

$$
|\mathbb{P}(\Gamma \cap \Theta \mid \Upsilon)-\mathbb{P}(\Gamma \mid \Upsilon) \mathbb{P}(\Theta \mid \Upsilon)| \mathbb{P}(\Upsilon)^{2}<2 \varepsilon
$$

Then,

$$
\begin{aligned}
& \mathbb{P}(\Gamma \cap \Theta)-4 \varepsilon \\
< & \mathbb{P}(\Gamma \cap \Theta)-\mathbb{P}(\Gamma \cap \Theta \mid \Upsilon) \mathbb{P}(\Upsilon)^{2}+\mathbb{P}(\Gamma \mid \Upsilon) \mathbb{P}(\Theta \mid \Upsilon) \mathbb{P}(\Upsilon)^{2}-2 \varepsilon \\
= & \mathbb{P}(\Gamma \cap \Theta)-\mathbb{P}(\Gamma \cap \Theta \cap \Upsilon) \mathbb{P}(\Upsilon)+\mathbb{P}(\Gamma \cap \Upsilon) \mathbb{P}(\Theta \cap \Upsilon)-\mathbb{P}(\Gamma) \mathbb{P}(\Theta)+\mathbb{P}(\Gamma) \mathbb{P}(\Theta)-2 \varepsilon \\
< & \mathbb{P}(\Gamma) \mathbb{P}(\Theta)
\end{aligned}
$$

In an analogous way it is shown that $\mathbb{P}(\Gamma) \mathbb{P}(\Theta)<\mathbb{P}(\Gamma \cap \Theta)+4 \varepsilon$. Hence, the result is proven.

Proposition 2 yields the proof of Theorem 11, by substituting $4 \varepsilon$ by $\varepsilon$. 


\section{Comparison of STIT and Poisson hyperplane tessellations (PHT)}

Intuitively, we expect a gradual difference in the mixing properties of STIT and PHT. Hyperplanes are unbounded, while the maximum faces (also referred to as I-faces) in a STIT tessellation are always bounded. But these I-faces tend to be very large (it was already shown in 10 for the planar case that the length of the typical I-segment has a finite expectation but an infinite second moment). But since we have shown that the tail $\sigma$-algebra for STIT is trivial, then STIT has a short range dependence in the sense of [2].

One aspect is the following. For PHT, Schneider and Weil [14 (Section 10.5) showed, that it is mixing if the directional distribution $\theta$ (see (22) ) has zero mass on all great subspheres of $\mathbb{S}_{+}^{\ell-1}$. And they contribute an example of a tessellation where this last condition is not fulfilled and which is not mixing. In contrast to this, Lachièze-Rey proved in 8$]$ that STIT is mixing, for all $\theta$ which are not concentrated on a great subsphere.

Concerning the tail $\sigma$-algebra, we have Theorem 2 for the STIT tessellations. In contrast, for the PHT the tail $\sigma$-algebra is not trivial.

Lemma 8 Let $Y^{\mathrm{PHT}}$ denote a Poisson hyperplane tessellation with intensity measure $\Lambda$ which is a non-zero, locally finite and translation invariant measure on $\mathcal{H}$, and it is assumed that the support set of $\Lambda$ is such that there is no line in $\mathbb{R}^{\ell}$ with the property that all hyperplanes of the support are parallel to it. Then the tail $\sigma$-algebra is not trivial with respect to the distribution of $Y^{\mathrm{PHT}}$.

Proof 10 Let $\left(W_{n}: n \in \mathbb{N}\right)$ be an increasing sequence of windows such that for all $n \in \mathbb{N}, W_{n} \subset \operatorname{int} W_{n+1}$, and $\mathbb{R}^{\ell}=\bigcup_{n \in \mathbb{N}} W_{n}$. Let $B_{1}$ be the unit ball in $\mathbb{R}^{\ell}$ centered at 0 . Consider the following event in $\mathcal{B}(\mathbb{T})$ :

$$
\mathcal{D}:=\left\{\exists \text { an hyperplane in } Y^{\mathrm{PHT}} \text { which intersects } B_{1}\right\} .
$$

Because outside of any bounded window $W_{n}$ all the hyperplanes belonging to $Y^{\mathrm{PHT}}$ can be identified and thus it can be decided (in a measurable way) whether there is a hyperplane which intersects $B_{1}$, we have that $\mathcal{D} \in \mathcal{B}\left(\mathbb{T}_{W_{n}^{c}}\right)$ for all $n \in \mathbb{N}$, and hence $\mathcal{D} \in \mathcal{B}_{-\infty}(\mathbb{T})$. We have $\mathbb{P}(\mathcal{D})=1-\mathrm{e}^{-\Lambda\left(\left[B_{1}\right]\right)}$, and this probability is neither 0 nor 1 .

Acknowledgments. The authors thank Lothar Heinrich for helpful hints and discussion. The authors are indebted for the support of Program Basal CMM from CONICYT (Chile) and by DAAD (Germany). 


\section{References}

[1] Breiman, L. (1993). Probability. 2nd ed., SIAM, Philadelphia.

[2] Daley, D.J. And Vere-Jones, D. (2008). An Introduction to the Theory of Point Processes. Vol. II: General Theory and Structure. 2nd ed., Springer.

[3] Heinrich, L. (1994). Normal approximation for some mean-value estimates of absolutely regular tessellations. Math. Methods Statist. 3, 1-24.

[4] Heinrich, L. (20012). Asymptotic methods in statistics of random point processes. in: E. Spodarav (ed.): Lectures on Stochastic Geometry, Spatial Statistics and Random Fields. Asymptotic Methods. Lecture Notes in Mathematics, Springer, ???-???.

[5] Heinrich, L. and Molchanov, I.S. (1999). Central limit theorem for a class of random measures associated with germ-grain models. Adv. Appl. Probab. 31, 283-314.

[6] Heinrich, L., Schmidt, H. and Schmidt, V. (2007). Limit theorems for functionals on the facets of stationary random tessellations. Bernoulli 13, 868-891.

[7] Kingman, J.F.C. (1992). Poisson Processes. Oxford University Press, Oxford.

[8] LachiÈze-Rey, R. (2011). Mixing properties for STIT tessellations. Adv. Appl. Probab. 43, 40-48.

[9] Martínez, S. and Nagel, W. (2012). Ergodic description of STIT tessellations. Stochastics: An Int. Journ. of Prob. and Stoch. Proc. 84, 113-134.

[10] Mecke, J., Nagel, W. And Weiss, V. (2007). Length distributions of edges of planar stationary and isotropic STIT tessellations. J. Contemp. Math. Anal. 42, 28-43.

[11] Mecke, J., Nagel, W. And Weiss, V. (2008). A global construction of homogeneous random planar tessellations that are stable under iteration. Stochastics 80, 51-67.

[12] Nagel, W. And Weiss, V. (2005). Crack STIT tessellations: Characterization of stationary random tessellations stable with respect to iteration. Adv. Appl. Probab. 37, 859-883.

[13] PARry, W. (2004). Topics in Ergodic Theory. Cambridge University Press. 
[14] Schneider, R. And Weil, W. (2008). Stochastic and Integral Geometry. Springer.

[15] Schreiber, T. and Thäle, C. (2010). Second-order properties and central limit theory for the vertex process of iteration infinitely divisible and iteration stable random tessellations in the plane. Adv. Appl. Probab. 42, 913-935.

[16] Schreiber, T. and ThäLe, C. (2011). Intrinsic volumes of the maximal polytope process in higher dimensional STIT tessellations. Stoch. Proc. Appl. 121, 989-1012.

[17] Schreiber, T. And ThäLe, C. (2012). Limit theorems for iteration stable tessellations. The Annals of Probability to appear. arXiv: math.PR/1103.3960 